\title{
EDITORIAL
}

\section{Is There Any Advantage of Using Polyclonal vs. Monoclonal Antibodies for Passive Enhancement of Immunity in Patients with COVID-19?}

On November 08, 2020, the US Food \& Drug Administration (FDA) approved the use of first monoclonal antibody - Bamlanivimab (LYCoV555; Eli Lilly \& Company) for the treatment of high-risk patients with mild-moderate SARS-CoV2 infection (1). Subsequently, on November 23, 2020, FDA approved another cocktail of monoclonal antibodies - Casirivimab + Imdevimab (REGEN-COV; Regeneron Pharmaceuticals) for use in a similar cohort of high-risk patients with mild-moderate COVID-19. Since the initial Emergency Use Authorizations (EUA), FDA has made numerous modifications/amendments including revoking the authorization for monotherapy with Bamlanivimab (3); addition of Etesevimab to Bamlanivimab monotherapy; and authorization of another monoclonal antibody Sotrovimab (Xevudy; Glaxo, Smith \& Kline) (4), The primary objective of these emergency authorizations was to treat patients early in the course of the disease in an non-hospitalized setting thus mitigating the progression of COVID-19 to a more severe stage that would necessitate hospitalization.

With the availability of these monoclonal antibodies, we at DHR Health proceeded to obtain approval of a prospective study from the DHR Health Institute for Research \& Development Institutional Review Board to treat high-risk patients with mild-moderate COVID-19. The first patient was treated on November 25, 2020, and since then, we have infused anti-SARS-CoV-2 monoclonal antibodies in over 580 patients with very satisfactory outcomes. This included both patients who had mild-moderate SARS-CoV-2 infection as well for post-exposure prophylaxis in COVID-19 negative subjects.

While effective, monoclonal antibodies have inherent advantages and disadvantages when used for therapeutic purposes. Produced in vitro from immortalized plasma cells, monoclonal antibodies have single specificity and affinity which, reduces the probability of cross reactivity (Figure 1). Due to batch-to-batch homogeneity, effectiveness of monoclonal antibodies is much more predictable, and they serve as an ideal candidate for use in diagnostic immunoassays. A distinct disadvantage of monoclonal antibodies is the fact that due to its high specificity and affinity, a minor mutation in the epitope often renders the antibody ineffective in neutralizing the target. This is a serious limitation since observed mutations in SARS-CoV-2 with generations of numerous variants may render the available monoclonal antibodies less effective in mitigating the progression of COVID-19. On the contrary, polyclonal antibodies are a mixture of monoclonal antibodies that are products of multiple B cell clones. Generated in animals, polyclonal antibodies recognize with reasonably high affinity multiple epitopes on a single antigen (Figure 1). This affords polyclonal antibodies a distinct advantage over monoclonal antibodies when used as therapeutic agents particularly in patients infected with rapidly mutating SARSCoV-2 virus.

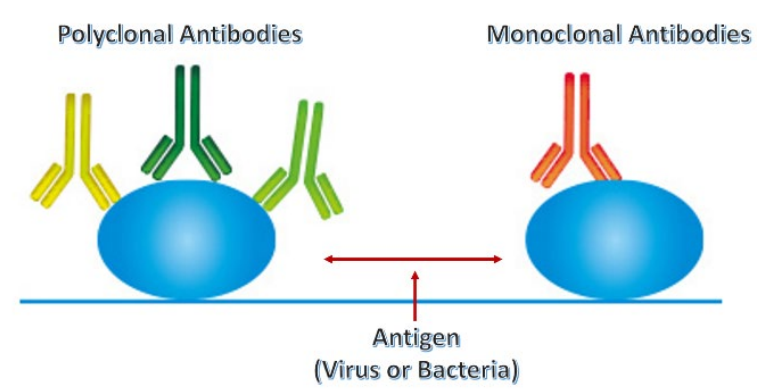

Figure 1: Monoclonal antibody have affinity for a single epitope on the antigen (i.e., the virus or the bacteria). On the contrary, polyclonal antibodies are a mixture of monoclonal antibodies with affinity for various epitopes on the antigen. 
On April 17, 2020, the National Institutes of Health (NIH) announced the Accelerating COVID-19 Therapeutic Interventions and Vaccines (ACTIV) public-private partnership to develop a coordinated research strategy for prioritizing and speeding development of the most promising treatments and vaccines. ACTIV was based on Bayesian adaptive clinical trial design which allows for frequent "looks" at the data and permits data-driven modifications during the course of the study (5). DHR Health Institute for Research and Development is part of the ACTIV collaborative and have initiated numerous studies in an attempt to find the most promising therapeutic agent to prevent and/or treat patients with COVID-19.

As part of ACTIV-2, DHR Health Institute for Research \& Development has initiated a prospective Phase $2 / 3$ clinical trial to evaluate the efficacy of fully human anti-SARS-CoV-2 polyclonal antibody (SAB-185; SAB Pharmaceuticals, Inc.) to treat unvaccinated nonhospitalized high-risk patients with mild-moderate COVID-19. SAB-185 is a fully human, anti-SARSCoV-2 immunoglobulin produced in transchromosomic bovines (Tc-hIgG-SARS-CoV2) hyperimmunized with two doses of plasmid DNA encoding the SARS-CoV-2 Wuhan strain S gene, followed by repeated immunization with $S$ protein purified from insect cells $(6,7)$. The resulting Tc-hIgG-SARS-CoV-2, termed SAB-185, efficiently neutralizes SARS-CoV-2, and vesicular stomatitis virus (VSV) SARS-CoV-2 chimeras in vitro. This fully human polyclonal antibody that potently inhibits SARS-CoV-2 infection may provide an effective therapeutic agent to combat COVID-19. This is not a placebo-controlled study as the patients in the comparator arm will be treated with REGEN-COV monoclonal antibody.

In conclusion, the use of available anti-SARS-CoV2 monoclonal antibodies has been effective in mitigating the progression of disease in high-risk patients with mild-moderate COVID-19. However, it could be argued that the use of fully human polyclonal antibodies with affinity for multiple epitopes on SARS-CoV-2 may be more effective in eliminating the virus and its variants which exhibit higher transmissibility, infectivity, and the potential to "escape" from the neutralizing effects of existing experimental agents.

\section{References}

1. Emergency Use Authorization (EUA) for bamlanivimab $700 \mathrm{mg}$ IV Center for Drug Evaluation and Research (CDER) Review. $2020 . \quad$ https://www.fda.gov/media/144118/download (Accessed October 26, 2021).

2. Emergency Use Authorization for REGEN-COV for the treatment of mild-moderate COVID-19. 2020. https://www.fda.gov/media/145610/download . (Accessed October 26, 2021).

3. FDA Revokes Initial Emergency Use Authorization of Monotherapy with Bamlanivimab. March 2021. https://www.fda.gov/media/143602/download (Accessed October 27, 2021)

4. FDA Authorizes Additional Monoclonal Antibody for Treatment of COVID-19. 2021. https://www.fda.gov/newsevents/press-announcements/coronavirus-covid-19-update-fdaauthorizes-additional-monoclonal-antibody-treatment-covid-19 (Accessed October 27, 2021)

5. Giovagnoli A. (2021). The Bayesian Design of Adaptive Clinical Trials. International journal of environmental research $\begin{array}{lll}\text { and public } & \text { health, } & \text { 18(2), }\end{array}$ https://doi.org/10.3390/ijerph18020530

6. Gilliland T, Liu Y, Li R, Dunn M, Cottle E, Terada Y, Rickman Z, Alcorn M, Vasilatos S, Lundy J, Larson D, Wu H, Luke T, Bausch C, Egland K, Sullivan E, Wang Z, Klimstra WB. Protection of human ACE2 transgenic Syrian hamsters from SARS CoV-2 variants by human polyclonal IgG from hyperimmunized transchromosomic bovines. bioRxiv [Preprint]. 2021 Jul 26:2021.07.26.453840. doi: 10.1101/2021.07.26.453840. PMID: 34341790; PMCID: PMC8328057.

7. Liu Z, Wu H, Egland KA, Gilliland TC, Dunn MD, Luke TC, Sullivan EJ, Klimstra WB, Bausch CL, Whelan SPJ. Human immunoglobulin from transchromosomic bovines hyperimmunized with SARS-CoV-2 spike antigen efficiently neutralizes viral variants. Hum Vaccin Immunother. $2021 \mathrm{Jul}$ 6:1-10. doi:10.1080/21645515.2021.1940652. Epub ahead of print. PMID: 34228597; PMCID: PMC8290372.

\section{Sohail Rao, MD, MA, DPhil}

Executive Vice President, DHR Health, 5501 S. McColl Road, Edinburg, Texas

President \& Chief Executive Officer, DHR Health Institute for Research \& Development, 5323 S. McColl Road, Edinburg, Texas

Corresponding author email: s.rao@dhr-rgv.com

Disclosures: None

ORCID: Sohail Rao: https://orcid.org/0000-0001-5027$\underline{9992}$ 


\section{Caitlin White}

DHR Health Institute for Research \& Development, 5323 S. McColl Road, Edinburg, TX 78539.

Disclosures: None

ORCID: Caitlin White: https://orcid.org/0000-00015528-2242

\section{Manish Singh, MD, FACS}

Chief Executive Officer, DHR Health, 5501 S. McColl Road, Edinburg, Texas

DHR Health Bariatric \& Metabolic Institute, 5500 Raphael Drive, Edinburg, TX 79539

Disclosures: None

ORCID: Manish Singh: https://orcid.org/0000-00034146-3282 